\title{
Adaptación al cautiverio de las especies; Anableps dowei, Poecilia gillii, Amatlitania nigrofasciata, Para el desarrollo de la acuariología en la zona sur de Honduras
}

Adaptation to the captivity of the species; Anableps dowei, Poecilia gilli, Amatlitania nigrofasciata, for the development of aquariology in the southnern zone of Honduras

DOI 10.5377pc.v0i16.8095

Cristhian Canales, Kaina Alvarado, Maury Carbajal ${ }^{1}$

Roberto Martínez, Liliam Carina Marroquín Tejeda ${ }^{2}$

\section{RESUMEN}

Se ha visualizado la acuariología como una alternativa para impulsar la innovación acuícola y lograr un mejor aprovechamiento de la biodiversidad acuática en la región, por lo que se planteó en este estudio evaluar la adaptación al cautiverio y la aceptación al alimento comercial de tres especies icticas con potencial ornamental, nativas del sur de Honduras; A. dowei, p. gilli y A. nigrofasciata. En el diseño de la investigación se contrastaron dos tratamientos, (pecera con sustrato natural y pecera con sustrato comercial) y tres repeticiones por tratamiento, de igual manera un tratamiento control sin sustrato, se estudiaron 49 organismos distribuidos en cada una de las peceras durante un periodo de seis semanas, en las que se monitoreo la sobrevivencia como indicador de adaptación. Con la misma frecuencia se midió el tiempo que le tomó a los organismos ingerir el alimento suministrado. Hechas las observaciones anteriores se determinó que las especies, A. nigrofasciata y P. gilli obtuvieron un $11 \%$ y un $22 \%$ respectivamente más de sobrevivencia en comparación con las peceras de sustrato comercial. En cambio $A$. dowei no sobrevivió en ningún tratamiento. Con respecto a la aceptación del alimento, los peces lo consumieron en un tiempo promedio de 26.13 segundos sin verse influenciada esta variable por el tipo de sustrato. Es importante mencionar que las mortalidades de A. dowei fueron ocasionadas por ataques de A. nigrofasciata dado que es una especie agresiva y territorial, Se estima que las especies se adaptaron al cautiverio independientemente del tipo de sustrato

1 Estudiantes de la carrera Ingeniería en Ciencias Acuícolas y Recursos Marino Costero, CURLP-UNAH: cristhian.canales@unah.hn; gisselaalvarado@unah.hn; maury.carvajal@unah.hn

${ }^{2}$ Asesores de investigación, Profesores de la carrera de Ingeniería en Ciencias Acuícolas y Recursos Marino Costeros CURLP-UNAH: robertomartinez@unah.edu.hn; liliam.marroquin@unah.edu.hn 
y de no ser por el factor comportamiento los porcentajes de sobrevivencia habrían sido significativos para todas las especies.

Palabras clave: Acuariología, Adaptación, especies ornamentales, Honduras.

\section{ABSTRACT}

Aquariology has been visualized as an alternative to promote aquaculture innovation and achieve a better use of aquatic biodiversity in the region, so it was proposed in this study to evaluate the adaptation to captivity and acceptance to the commercial feed of three fish species with ornamental potential, native to southern Honduras; A. dowei, p. gilli and A. nigrofasciata. In the design of the research, two treatments were compared (fish tank with natural substrate and a fish tank with commercial substrate) and three repetitions per treatment, in the same way a control treatment without substrate, and 49 organisms distributed in each of the fish tanks were studied during a period of six weeks, in which survival was monitored as an indicator of adaptation. With the same frequency, the time it took the organisms to eat the food supplied was measured. Based on the previous observations, it was determined that the species, $P$. gilli and $\mathrm{A}$. nigrofasciata obtained $11 \%$ and $22 \%$ more survival in comparison with commercial substrate fish tanks. In contrast A. dowei did not survive in any treatment. Regarding the acceptance of the food, the fish consumed it in an average time of 26.13 seconds without this variable being influenced by the type of substrate. It is important to mention that the deaths of A. dowei were caused by attacks of A. nigrofasciata since it is an aggressive and territorial species. It is estimated that the species adapted to captivity regardless of the type of substrate and if not for the behavioral factor, the survival percentages would have been significant for all species.

Keywords: Acuariology, Adaptation, ornamental species, Honduras. 


\section{INTRODUCCIÓN}

La acuacultura ha tenido un desarrollo muy relevante en Honduras, en el año 2016 la camaronicultura representó el tercer rubro de mayor exportación registrando según la FAO 2016, 202.22 millones de dólares anuales siendo un $21.24 \%$ mayores que los ingresos de 2015. Dicho desarrollo ha sido impulsado en la zona sur del país, cuya producción se concentra en el área del Golfo de Fonseca. Otra industria fundamental es la piscicultura, dedicada está última al cultivo de tilapia (Sotorrío, 2006).

A pesar del gran aporte a la economía del país, que generan las exportaciones de las especies cultivadas tradicionalmente (Litopenaeus vannamei) (Oreochromis Sp), es necesario diversificar los cultivos y ampliar los campos de estudio de la acuacultura, una alternativa viable (debido a los ingresos que genera y a su auge durante los últimos años) es la piscicultura ornamental también conocida como acuariología 0 acuariofilia, misma que ha tenido crecimiento en el país con el uso de peces ornamentales exóticos para fines recreativos y decorativos, no obstante la reproducción y crianza de peces ornamentales es un tema nuevo en el país y poco conocido por los piscicultores.

De igual manera dentro de un contexto local se tiene muy poco conocimiento respecto al tema ya que la producción acuícola en la región sur de Honduras se ha fijado únicamente como objetivo la producción de especies acuáticas para el consumo, en la actualidad no se contempla la cría con fines decorativos.

Sobre la base de investigaciones anteriores en el país, se puede concluir que en Honduras la industria del acuario se ha desarrollado únicamente con especies exóticas, todavía no se han registrado investigaciones que desarrollen la acuariología empleando especies nativas.

A pesar de que las condiciones geográficas del territorio nacional son idóneas para el desarrollo de la industria por su biodiversidad, con la presencia de 172 especies que habitan en las aguas del país, de las cuales 166 son nativas y seis exóticas (Matamoros, 2009). Estas especies en su mayoría no han sido estudiadas teniendo como finalidad la cría ornamental.

Debido a la biodiversidad y al potencial acuícola con el que cuenta esta región, se propone implementar alternativas innovadoras para ampliar el campo de la investigación científica en el rubro de la acuacultura, y de esta manera ejecutar un mejor apro- 
vechamiento de los recursos acuáticos, por lo que se propone la acuariología como alternativa viable de producción y comercialización en el contexto local y nacional.

Actualmente la industria de la acuariofilia es un mercado que se encuentra en expansión. A un cuando no existe información exacta respecto a cifras, se calcula que esta industria genera ganancias sobre US\$300 millones con una tasa de crecimiento anual del 14\% (Reynoso, 2012).

Consecuente a su viabilidad y a la disponibilidad de recursos se planteó esta investigación que tuvo como objetivo la adaptación al cautiverio de especies locales de la zona sur de Honduras (Anableps dowei, Poecilia gilli, Amatitlania nigrofasciata) para el desarrollo de la acuariología, de manera que se estudió su capacidad para sobrevivir en distintos tipos de sustrato (sustrato Natural y Comercial). así como su aceptación al alimento comercial para especies tropicales.

con el fin de obtener conocimientos que permitan implementar técnicas y métodos que sirvan de guía para poder incursionar por primera vez en el desarrollo de esta industria, hasta hoy inexplorada en la región.

Misma que cuenta con una perspectiva de crecimiento social y económico, ya que esta representa una importante derrama económica de ingresos que contribuyen a mejorar la economía de quienes se dedican a ella, convirtiéndola en una actividad muy distintiva y rentable (Rodriguez, 2006)

\section{MATERIALES Y MÉTODO}

\section{Delimitación del objeto de estudio}

La Investigación Se realizó en las instalaciones del laboratorio Húmedo CURLP-UNAH (13.327372, -87.135590). 5km Salida a San Marcos, la misma consistió en evaluar durante seis semanas (26 de febrero al 9 de abril del 2018) la adaptación al cautiverio en peceras de las especies en estudio, expuestas a diferentes sustratos.

\section{Captura de las especies}

Se capturaron con una atarraya de $7 \mathrm{~m}^{2}, 21$ ejemplares de P. gilli y siete de A. dowei 
en el Río Choluteca, Choluteca y 21 organismos de la especie A. nigrofasciata en la comunidad de agua zarca san Lorenzo, Valle. En los puntos

1: 13. $18657,-87.11526$

2: $13.437918,-87.368479$

llustración 1. Ubicación de los puntos para la toma de muestras. (Google maps).

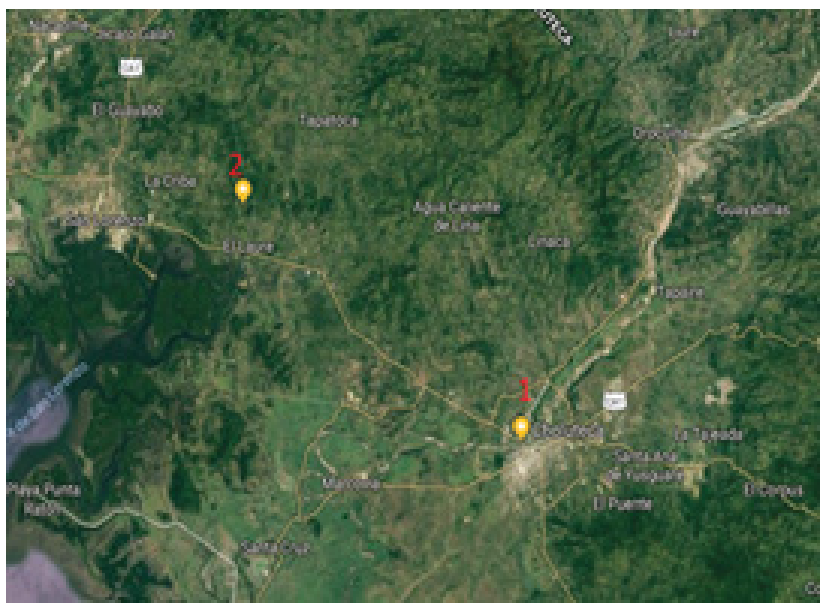

\section{Transporte}

Los peces se transportaron en tres baldes con capacidad para 50 litros de agua cada uno, y para mantener los niveles de oxígeno se utilizó un proveedor portátil.

\section{Aclimatación}

El proceso de aclimatación de los peces se desarrolló tomando como punto de referencia la temperatura del agua a la que fueron capturados y la temperatura en la que se encontraba el agua potable del laboratorio húmedo. Para lograr que los organismos asimilaran el cambio de temperatura de manera gradual se reguló el contraste entre las dos temperaturas vertiendo agua del laboratorio cada 15 minutos al medio de los peces para lograr aumentar la temperatura, regulando los grados uno a uno.

\section{Periodo de observación}

Una vez aclimatados a la temperatura del agua se dejaron las especies individualmente en observación durante seis días. 


\section{Instalación}

Se instalaron siete peceras en un estante de madera y bases de hierro con capacidad para albergar 35 litros de agua potable, de las cuales tres contenían sustrato comercial (SA1, SA2, SA3) y tres sustratos naturales (SN1, SN2, SN3), así mismo se instaló una pecera sin sustrato que se utilizó como prueba control (PC).

\section{Tratamiento antibacteriano}

Se aplicaron $1.5 \mathrm{ml}$ de oxitetraciclina en $35 \mathrm{I}$ de agua durante 24 horas para tratar los peces que presentaban síntomas de infección bacteriana.

\section{Composición del sustrato}

Se utilizaron 4 libras de sustrato comercial de grava semi-fina, de igual manera tres plantas artificiales en cada una de las peceras destinadas a este tipo de sustrato. En cuanto a las peceras que contendrían sustrato natural se utilizaron 2 libras de arena tomada del río en que se capturaron los organismos así mismo cuatro troncos de madera.

\section{Introducción de las especies al medio}

Una vez instaladas las peceras se procedió a introducir los organismos en las peceras de manera que las tres especies en estudio convivieran entre sí.

\section{Calidad del agua}

Se monitoreo la calidad del agua tres veces por semana tomando en cuenta parámetros como la temperatura, oxígeno disuelto, y contenido de amonio.

\section{Recambios de agua}

Se realizaron Recambios semanales de $20 \%$, y recambios cada 15 días del $100 \%$ del total del agua en las peceras.

\section{Alimentación}

Se suministró alimento comercial para especies tropicales en escamas en dos raciones diarias una por la mañana y otra por la tarde, y se midió con un cronometro de 
mano el tiempo en que los organismos lo ingerían.

\section{Diseño experimental}

Se utilizó un diseño en bloques con 2 tratamientos (Sustrato Natural y Comercial) y tres repeticiones por tratamiento, de igual manera un tratamiento control, las unidades experimentales fueron A. nigrofasciata, P. gilli, A. dowei.

En ambos tratamientos se suministró alimento comercial para especies tropicales Los datos obtenidos de sobrevivencia y tiempos de alimentación se analizaron en hojas de Excel.

\section{RESULTADOS}

\section{Sobrevivencia}

Mediante comparación de tratamiento se determinó que las especies A. nigrosfaciata y P. gillii obtuvieron un $11 \%$ y un $22 \%$ más de sobrevivencia en comparación con las peceras de sustrato comercial.

\section{Ilustración 2. Diseño experimental}


\section{Elaboración: fuente propia}


Tabla 1. Porcentaje de sobrevivencia de cada especie en cada tratamiento

\begin{tabular}{|c|c|c|c|}
\hline Sobrevivencia & A. nigrofasciata & P. gilli & A. dowei \\
\hline SA & $33 \%$ & $22 \%$ & $0 \%$ \\
\hline SN & $44 \%$ & $44 \%$ & $0 \%$ \\
\hline PC & $67 \%$ & $0 \%$ & $0 \%$ \\
\hline
\end{tabular}

Elaboración: fuente propia

\section{Mortalidad}

Durante la primera semana de estudio hubo mortalidad de la especie A. dowei en todas las peceras, así mismo ocurrieron muertes graduales de las otras especies viéndose afectados cinco individuos de A. nigrofasciata de los cuales dos murieron en cada uno de los sustratos y uno en la prueba control y de la especie P. gilli tres organismos de sustrato artificial y uno en sustrato natural.

Gráfico 1. Cantidad de organismos muertos en las seis semanas del experimento

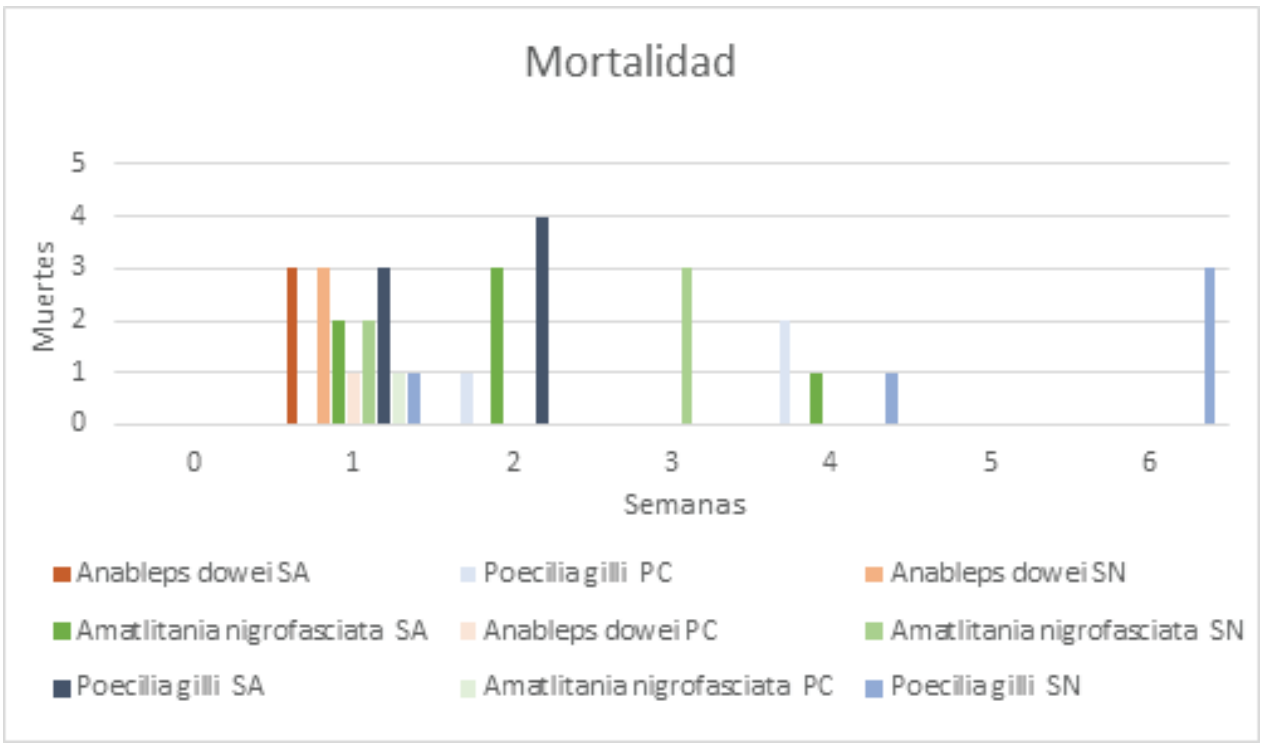

Elaboración: fuente propia 


\section{Aceptación del alimento comercial}

Los peces se alimentaron en la jornada matutina y vespertina con una diferencia de 10 y 15 segundos respectivamente en lo que respecta al tiempo transcurrido desde la primera semana hasta la última. La cantidad de alimento que cada pez consumió en una semana fue de 0.20 gramos, durante las dos jornadas de alimentación los peces consumieron el alimento en un tiempo promedio de 26.13 segundos.

\section{Gráfico 2. Tiempo de ingesta del alimento.}

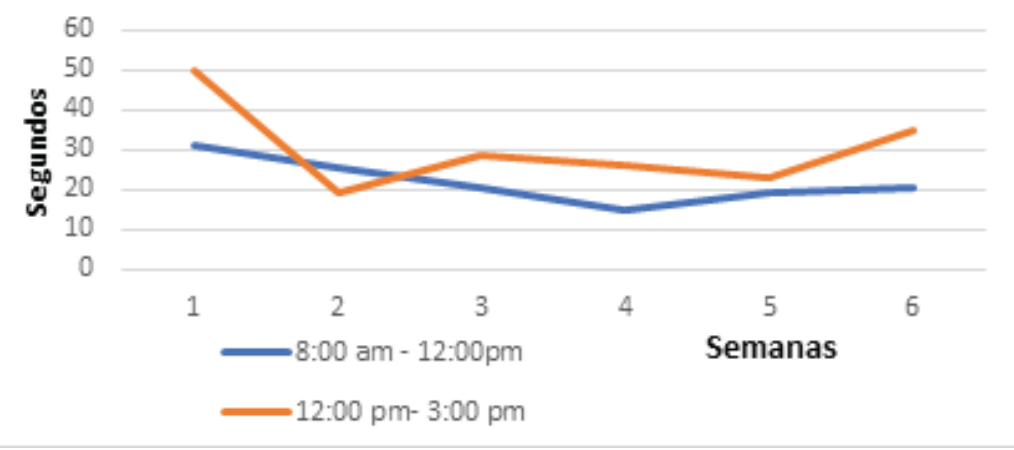

Elaboración: fuente propia

\section{Parámetros}

En los monitores de calidad de agua durante la investigación se registraron en promedio los valores que se presentan.

\section{Gráfico 3. Monitoreo de parámetros}



Elaboración: fuente propia 
Los parámetros se mantuvieron dentro de los rangos aceptables para los objetivos del estudio, durante las seis semanas las peceras con sustrato natural presentaron una diferencia de $0.31 \mathrm{mg} / \mathrm{l}$ de amoniaco menos con respecto a las peceras de sustrato comercial, la prueba control se mantuvo dentro de un rango de $0.63 \mathrm{mg} / \mathrm{l}$, mostrando una diferencia de 0.26 y $0.57 \mathrm{mg} / \mathrm{l}$ más en sustrato comercial y natural respectivamente.

\section{DISCUSION}

Para la sobrevivencia resulta oportuno mencionar que la especie A. nigrosfaciata es agresiva y territorial por lo que ataco a muerte a la especie A. dowei en la primera semana de estudio eliminando totalmente su población, las muertes se le atribuyen a esta ya que se observó en varias ocasiones como les seguían y mordían su parte ventral, así mismo, debido a que en la semana de observación cuando las especies se encontraban separadas, los individuos se mostraron activos y saludables en cautiverio.

En cuanto al tipo de sustrato se determinó que hubo una mayor sobrevivencia para las especies A. nigrofascaiata y P. gilli en las peceras con sustrato natural, esto como consecuencia de la función de refugio que posee el sustrato natural, lo que permitía que los peces se mantuvieran seguros cuando se sentían amenazados por los individuos agresivos.

El $85 \%$ de la población se redujo por ataques de A. nigrofasciata a su misma especie y a organismos de p. gilli, el $15 \%$ de mortalidades restantes fueron ocasionadas por cambios de temperatura no previstos a los que fueron expuestos los organismos de P. gilli al momento de hacer recambios de agua, esto como consecuencia de un cambio brusco de temperatura de $28^{\circ} \mathrm{C}$ a $35^{\circ} \mathrm{C}$ el cual no resulto tolerable para algunos individuos de la especie.

Cabe resaltar que en las peceras donde hubo una dominancia de machos de A. nigrofasciata se presentaron las mayores mortalidades, contrario a lo que ocurrió en las peceras en donde había una distribución de dos hembras un macho o dominancia de hembras.

Las variaciones en los segundos de ingesta del alimento comercial se dieron debido a que en las primeras semanas el tiempo que le tomaba a los organismos ingerir y 
asimilar el alimento era mayor ya que no formaba parte de su dieta natural, conforme pasaron las semanas los individuos se alimentaron en un menor tiempo, aceptando cada vez más el alimento, lo que los llevo al desarrollo de nuevos hábitos y comportamientos alimenticios distintos a los de su hábitat natural.

Las variaciones en los niveles de amoniaco se le atribuyen al tipo de sustrato, en vista de que las peceras con sustrato natural poseían una menor cantidad que el tratamiento con sustrato comercial, esto debido a la capacidad filtradora que tiene la arena, lo que permitía que estas peceras se mantuvieran con niveles más bajos de amoniaco, con aguas más claras y apariencia estética agradable, por otra parte la pecera que carecía de sustrato obtuvo el más alto nivel de amoniaco superando por una cantidad considerable a las peceras con sustrato natural.

\section{CONCLUSIONES}

De acuerdo con los resultados obtenidos las especies se adaptaron satisfactoriamente al cautiverio independientemente del tipo de sustrato; de no ser por la agresividad de la especie antes mencionada la sobrevivencia en las peceras habría sido significativa para todos los organismos, por lo que para estudios posteriores será preciso considerar el comportamiento de las especies y la distribución de sexos por pecera para disminuir la agresividad de los individuos.

En cuanto a la aceptación del alimento las especies ingerían el total del alimento suministrado sin verse afectada esta variable por el tipo de sustrato.

\section{RECOMENDACIONES}

Considerar la distribución de sexos y tamaños de los peces en el acuario, de modo que haya una dominancia de hembras o una distribución en parejas.

Colocar refugios artificiales en el acuario, que les permitan a los peces introducirse en ellos ya sea para protegerse a sí mismos o para conservar energías.

No introducir más 2 individuos de A. nigrofascaita en una pecera con capacidad para 35 litros de agua 


\section{REFERENCIAS BIBLIOGRÁFICAS}

Fao. (2015). Perfiles de la pesca y la acuicultura por paises- Honduras. Obtenido de http://www.fao.org/fishery/facp/HND/es

Fisheries FAO. (2016). fisheries. Obtenido de Colecciones de estadísticas de pesca

Reynoso, F. L. (2012). La acuariofilia de especies ornamentales marinas: un mercado de retos y oportunidades. Scielo, 12-21.

Rodriguez, F. (2006). Acuarofilia Rentable.

Sotorrio, L. (2006). Estudio del sector acuicola en paises Lationoamericanos: Honduras. R. D. Wilfredo A. Matamoros, J. F. (2009). Annotated cheklist of the frshwater fishes of continental and insular Honduras. mapress.com, 1-38. 